\title{
Femtosecond-Laser-Based Inscription Technique for Post-Fiber-Bragg Grating Inscription in an Extrinsic Fabry-Perot Interferometer Pressure Sensor
}

Sven Poeggel, Dineshbabu Duraibabu, Amedee Lacraz, Kyriacos Kalli, Daniele Tosi, Gabriel Leen, Elfed Lewis

- School of Engineering

\begin{abstract}
In this paper, a novel fiber Bragg grating inscription technique based on a femtosecond laser is presented. The grating was inscribed in close proximity to the tip of an extrinsic Fabry-Perot interferometer (EFPI)based optical fiber pressure sensor. This therefore represents an optical fiber pressure and temperature sensor (OFPTS) for simultaneous pressure and temperature measurement for use in exactly the same physical location. The temperature measurement can also be used to compensate thermal drift in the EFPI sensor. The Bragg wavelength can be tailored precisely to any given wavelength in the optical spectrum and the degree of reflection can be adjusted to suit the FPI spectrum. The OFPTS has a diameter of 200 $\$ \backslash m u \backslash$ text $\{m\} \$$ and is fully biocompatible. Furthermore, the sensor shows a high stability after grating inscription, of better than $0.5 \%$ in $20 \mathrm{~min}$. The small size and high stability makes the sensor especially interesting for volume restricted areas, like blood vessels or the brain.
\end{abstract}

Original language $\quad$ English

Article number 7109831

Pages (from-to) 3396-3402

Number of pages 7

Journal IEEE Sensors Journal

Volume 16

Issue number $\quad 10$

State $\quad$ Published - May 152016

Poeggel, S., Duraibabu, D., Lacraz, A., Kalli, K., Tosi, D., Leen, G., \& Lewis, E. (2016). Femtosecond-LaserBased Inscription Technique for Post-Fiber-Bragg Grating Inscription in an Extrinsic Fabry-Perot Interferometer Pressure Sensor. IEEE Sensors Journal, 16(10), 3396-3402. [7109831]. DOI: 10.1109/JSEN.2015.2434772 